\title{
BMJ Open Reducing COVID-19 quarantine with SARS-CoV-2 testing: a simulation study
}

To cite: Peng B, Zhou W, Pettit RW, et al. Reducing COVID-19 quarantine with SARS-CoV-2 testing: a simulation study. BMJ Open 2021;11:e050473. doi:10.1136/ bmjopen-2021-050473

- Prepublication history and additional supplemental material for this paper are available online. To view these files, please visit the journal online (http://dx.doi.org/10.1136/ bmjopen-2021-050473)

Received 25 February 2021 Accepted 29 April 2021

Check for updates

(C) Author(s) (or their employer(s)) 2021. Re-use permitted under CC BY-NC. No commercial re-use. See rights and permissions. Published by BMJ.

${ }^{1}$ Institute for Clinical \& Translational Research, Baylor College of Medicine, Houston, Texas, USA

${ }^{2}$ Corporate Medical Advisors, Houston, Texas, USA

${ }^{3}$ Department of Laboratory

Medicine, University of

Washington, Seattle,

Washington, USA

${ }^{4}$ International SOS, Houston, Texas, USA

Correspondence to Dr Christopher I Amos; chrisa@bcm.edu

\author{
Bo Peng, ${ }^{1}$ Wen Zhou, ${ }^{1}$ Rowland W Pettit, ${ }^{1}$ Patrick Yu, ${ }^{2}$ Peter G Matos, ${ }^{2}$ \\ Alexander L Greninger, ${ }^{3}$ Julie McCashin, ${ }^{4}$ Christopher I Amos (i) ${ }^{1}$
}

\begin{abstract}
Objective To evaluate the effectiveness of SARS-CoV-2 testing on shortening the duration of quarantines for COVID-19 and to identify the most effective choices of testing schedules.

Design We performed extensive simulations to evaluate the performance of quarantine strategies when one or more SARS-CoV-2 tests were administered during the quarantine. Simulations were based on statistical models for the transmissibility and viral loads of SARSCoV-2 infections and the sensitivities of available testing methods. Sensitivity analyses were performed to evaluate the impact of perturbations in model assumptions on the outcomes of optimal strategies.
\end{abstract}

Results We found that SARS-CoV-2 testing can effectively reduce the length of a quarantine without compromising safety. A single reverse transcription-PCR (RT-PCR) test performed before the end of quarantine can reduce quarantine duration to 10 days. Two tests can reduce the duration to 8 days, and three highly sensitive RT-PCR tests can justify a 6-day quarantine. More strategic testing schedules and longer quarantines are needed if tests are administered with less-sensitive RT-PCR tests or antigen tests. Shorter quarantines can be used for applications that tolerate a residual postquarantine transmission risk comparable to a 10-day quarantine.

Conclusions Testing could substantially reduce the length of isolation, reducing the physical and mental stress caused by lengthy quarantines. With increasing capacity and lowered costs of SARS-CoV-2 tests, test-assisted quarantines could be safer and more cost-effective than 14-day quarantines and warrant more widespread use.

\section{INTRODUCTION}

Until herd immunity to COVID-19 develops through infection or vaccination, quarantine will remain the primary means of disease mitigation. The US Centers for Diseases Control and Preventions (CDC) currently recommends a 14-day prophylaxis quarantine for anyone who comes into contact with a person who has COVID-19. ${ }^{1}$ The 14-day quarantine recommendation is based on clinical data of the observed incubation period. ${ }^{2}$ Projections indicated that a 14-day quarantine period sequesters $99 \%$ of individuals who have been exposed and would ultimately develop COVID-19.
Strengths and limitations of this study

- We developed a novel forward-time simulation ap proach allowing the modelling of individual characteristics and complex quarantine and testing approaches.

- Our simulations indicate that the 14-day quarantine approach is overly conservative and can be safely shortened if testing is performed.

- Our recommendations include testing schedules that could be immediately adopted and implemented as government and industry policies.

- Results depend on known characteristics of SARSCoV-2 tests and population characteristics of transmission.

The CDC guideline on a 14-day quarantine has been adopted globally by most organisations and government agencies. Government agencies use quarantine after travel to reduce the introduction of potentially infected individuals into communities. Industry and organisations, such as professional sports teams and movie productions, have used quarantine to produce a COVID-19-free cohort to resume operations, as mandated by most governments.

Currently, jurisdictions are starting to loosen quarantine requirements due to perceived lower risk of infection, negative psychological effects caused by lengthy quarantines, ${ }^{3}$ the costs of managing quarantines, loss of productivity and the need to ensure continuity of operations of essential functions of critical infrastructures. For example, the state of New York announced on 31 October 2020, new COVID-19 travel guidelines that allow travellers to exit quarantine after they test negative 4 days postarrival if they also test negative 3 days prior to departure. As of 11 September 2020, the CDC's guidance allows critical infrastructure workers to return to work without undergoing quarantine following workplace exposure. The European CDC (ECDC) published revised quarantine requirements allowing quarantine to be discontinued if the result from a SARS-CoV-2 
reverse transcription-PCR (RT-PCR) test is negative on day 10 after the last exposure with the warning of 'residual risk' for ending quarantine early. On 2 December 2020, CDC released a guideline on options to reduce quarantine for contacts of persons with SARS-CoV-2 infection using symptom monitoring and diagnostic testing. The policy allows shortening quarantine to 10 days without testing or 7 days with a SARS-CoV-2 test before release under the conditions of continued monitoring through day 14. The residual postquarantine transmission risk (PQTR) for a 7-day quarantine with RT-PCR testing was estimated to be $4.0 \%$ with a range between $2.3 \%$ and $8.6 \%$, based on currently unpublished results ${ }^{45}$

The recommendations from the European and US CDCs reflect both the benefits and uncertainties related to test-assisted quarantine. SARS-CoV-2 RT-PCR was initially developed for diagnostic purposes and later adopted for widespread screening of asymptomatic populations. As RT-PCR can detect very low viral loads, testing individuals before and during quarantine could identify individuals with subclinical disease before being infectious, improving quarantine efficacy. Faster and less-sensitive antigen tests could potentially also be used during quarantine. These methods were not recommended to the public at the early stages of the pandemic due to insufficient testing capacity and a lack of rigorous assessment of whether testing can sufficiently compensate for the elevated risks related to shortened quarantines. A few recent but nonpeer-reviewed studies indicate the benefits of COVID-19 tests during quarantine. ${ }^{4-6}$

Nevertheless, test-assisted quarantine has been performed, especially for industries where the benefits of shortened quarantine outweigh the costs of SARS-CoV-2 testing. For example, for movie productions, we have previously found a quarantine strategy with a 3-day pretravel quarantine and a 5-day posttravel quarantine with RT-PCR testing provides minimal risk for employees who are forced to work closely together. For individuals who arrived late or had to travel and rejoin the production, more rapid clearance protocols included daily or two times per day testing for 5 days were recommended. The proposed strategy for movie production was accepted by the cities of Chicago and Atlanta but denied by other governmental agencies. The recommendation and reservations of ECDC's guidelines and the mixed responses from governmental agencies support the need for a thorough investigation of the risks associated with test-assisted quarantine strategies and the potential to use tests with lower sensitivity, such as antigen testing, for managing cohorts of individuals undergoing quarantines.

Using an individual-based forward-time simulation method, ${ }^{7}$ we investigated the potential use of SARS-CoV-2 tests to implement a shorter and more effective quarantine strategy. Based on high-sensitivity RT-PCR results, we identified situations where a substantially shorter quarantine period is adequate to mitigate risk for assembling cohorts of protected individuals who will be closely interacting. We recommend guidelines for test-assisted quarantines using widely used testing methods and tests with well-characterised analytic and clinical sensitivities, for example, RT-PCR and rapid antigen tests. We also assessed factors that could affect the performance of test-assisted quarantines via simulation. Here, analytical sensitivity refers to the smallest amount of analyte (SARS-CoV-2 virus), in which a SARS-CoV-2 test will be positive for $95 \%$ of the time, that is, the limit of detection (LOD) and clinical sensitivity refers to the proportion of positive index tests in patients who have the disease in question. We noted that clinical sensitivity could vary due to human, specimen and collection factors, even for the same test kits.

\section{METHODS}

\section{Simulation method}

We used a COVID-19 outbreak simulator to simulate the quarantine of a large number of individuals of varying infectivity patterns. ${ }^{7}$ While the simulator was designed to simulate the spread of the SARS-CoV-2 virus in a population, we modified it to simulate the quarantine of a large number of individuals, compare the safety of various quarantine strategies and identify approaches with the shortest quarantine times and least number of tests. We ignored uninfected individuals in our simulations because they do not help evaluate the performance of quarantine strategies. An advantage of this approach was that it did not require any specific analytical form to evaluate viral spread and how interventions could affect transmission.

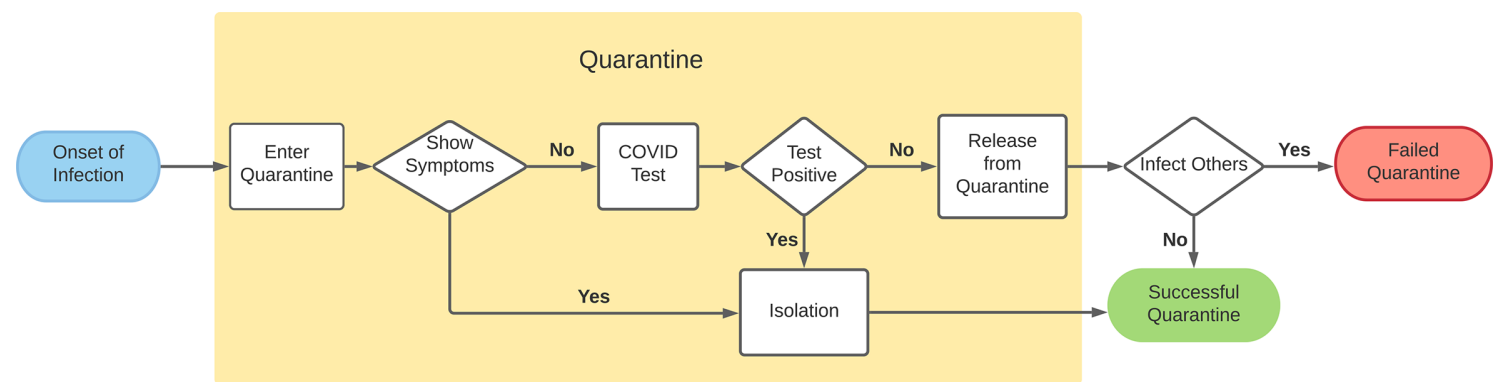

Figure 1 Diagram of a quarantine process with one test performed before the end of quarantine. In our simulations zero, one or more tests could be performed, and the carrier could show symptoms any time before the end of quarantine and be removed. 
Table 1 Summary of assumptions for the progression and infectivity of SARS-CoV-2 infection

\begin{tabular}{lll}
\hline Model & Parameter & Value \\
\hline Symptomatic & R0 & Normal $(2.10,0.36)$ \\
& $\begin{array}{l}\text { Incubation period } \\
\text { Duration of infection } \\
\text { after onset of }\end{array}$ & 2ogNormal $(1.62,0.42)$ \\
& symptoms & \\
& Infectivity starts & $1 / 5$ of incubation period \\
& Infectivity peaks & $2 / 3$ of incubation period \\
Asymptomatic & Ro & Normal $(0.42,0.07)$ \\
& Duration of infection & $3+$ LogNormal $(0.97,0.50)$ \\
& Infectivity starts & $1 / 10$ of duration of infection \\
& Infectivity peaks & $4 / 10$ of duration of infection \\
\hline
\end{tabular}

Models used to model the progression and infectivity of SARSCoV-2 infection for symptomatic and asymptomatic cases. The communicable period of symptomatic cases includes an incubation period modelled by a log normal distribution, and a period of infection after onset of symptoms, which is modelled by a shifted log normal distribution. The communicable period of asymptomatic carriers follows a shifted log normal distribution. Transmissibility probabilities are modelled by piecewise linear functions with a period with no infectivity, followed by a period with increasing infectivity, and then a period of decreasing infectivity.

As depicted in figure 1, simulated individuals were infected with the SARS-CoV-2 virus either right before entering quarantine or earlier but were asymptomatic when they entered quarantine. Using stochastic models for viral load and transmissibility described below and summarised in table 1, we simulated the viral load and transmissibility throughout the course of infection. Furthermore, we simulated the times when individuals become infectious, show symptoms if symptomatic, infect others within or outside the quarantine period and recover (ie, are no longer contagious but could have a detectable viral load). SARS-CoV-2 tests of specified sensitivity, specificity and turnaround time were applied, and individuals who tested positive or showed symptoms during the quarantine were removed from the simulation. We estimated PQTR from the proportion of simulations in which the individual completed quarantine and caused one or more infection events after being released.

Differing strategies may be needed for specific quarantine applications. We simulated scenarios in which individuals are quarantined after either simultaneous infection or exposure to SARS-CoV-2 or after infection at mixed intervals but without symptoms. These scenarios correspond to two major quarantine applications: (1) quarantine of a group of people after simultaneous exposure to a common source of potential infection, for example, shared close contact with an infected individual or participation in an event with known SARS-CoV-2positive attendees and (2) prequarantine of individuals with an unknown stage of infection before their assembly event, for example, sporting events, business meetings.

\section{Model of transmissibility}

A transmissibility model determines how many and when infected individuals transmit SARS-CoV-2 to others. We modelled the transmissibility of infected individuals using a piecewise function that starts with a period of noninfectivity, continues with a period of increasing infectivity and concludes with a period of declining infectivity. The overall transmissibility (ie, the probability of transmissibility integrated over the course of infection) was equal to the reproduction number $\left(\mathrm{R}_{\mathrm{e}}\right)$ of the infected individual (figure 2A).

The $R_{e}$ is the average number of secondary infections produced by an infectious person in a population. It can vary from population to population and in time based on demographics, public awareness, self-protection and possibly viral strain. ${ }^{8}$ Estimates of COVID- $19 \mathrm{R}_{\mathrm{e}}$ can be as high as $6.47^{10}$ and below 1 in many parts of the world. We modelled individual infectiousness with a biological component that relates to viral load and a social component that is impacted by factors such as social distancing and mask wearing. Viral load was simulated as base reproduction numbers drawn from a normal distribution with a 95\% CI between 1.4 and 2.8 for symptomatic cases and $0.28-0.56$ for asymptomatic carriers. ${ }^{11}$ Social determinants of infectivity were simulated through a distancing factor that, for example, doubles the base reproduction number if a simulated carrier will interact with others intensely without social distancing after quarantine. The overall reproduction number for the population depends on the proportion of individuals who are asymptomatic carriers. The proportion of individuals who are asymptomatic carriers was drawn from a normal distribution with $95 \%$ CI of 0.1 and $0.4,{ }^{12}$ so, on average, $25 \%$ of simulated individuals did not show any symptoms.

Infected individuals underwent an incubation period that followed a lognormal distribution with a mean of 5.5 days. ${ }^{2}$ Most presymptomatic transmission exposure occurs 1-3 days before a person develops symptoms, and viral loads are already at their peak or declining at the onset of symptoms. ${ }^{13-15}$ Therefore, we allowed transmission probabilities to start 1-2 days after infection, peak before the onset of symptoms and decline afterward (figure 2A). We set the period of infectivity after the onset of symptoms to follow a shifted lognormal distribution, so $88 \%$ and $95 \%$ of individuals are no longer infectious after 10 and 15 days, respectively. ${ }^{14}$ The transmissibility curves for asymptomatic carriers were similar, although the overall communicable periods (periods in which carriers stay infectious) were shorter than for symptomatic cases. ${ }^{16} 17$

\section{Model of viral load}

Transmission of the SARS-CoV-2 virus is caused by viral shedding from infected individuals, and the probability of infecting other individuals is related to the viral load of the infector. Viral shedding begins 5-6 days before the appearance of symptoms and decreases monotonically after symptom onset. ${ }^{18}$ The mean viral shedding duration is quite long, approximately 18 days for upper respiratory 
A

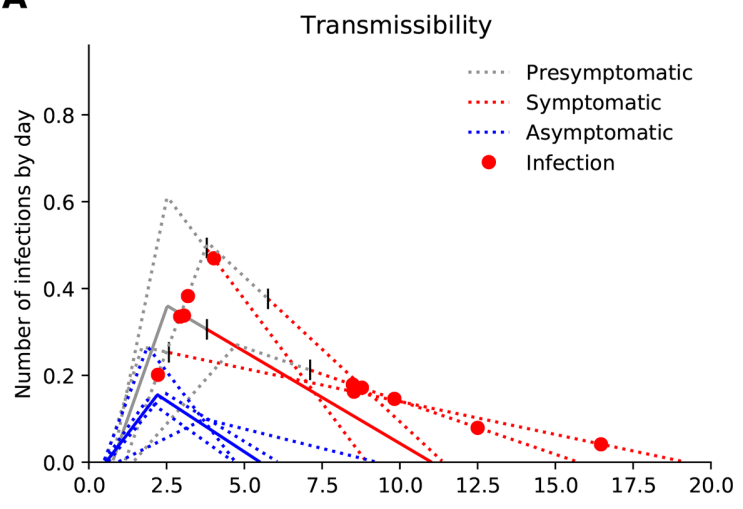

B

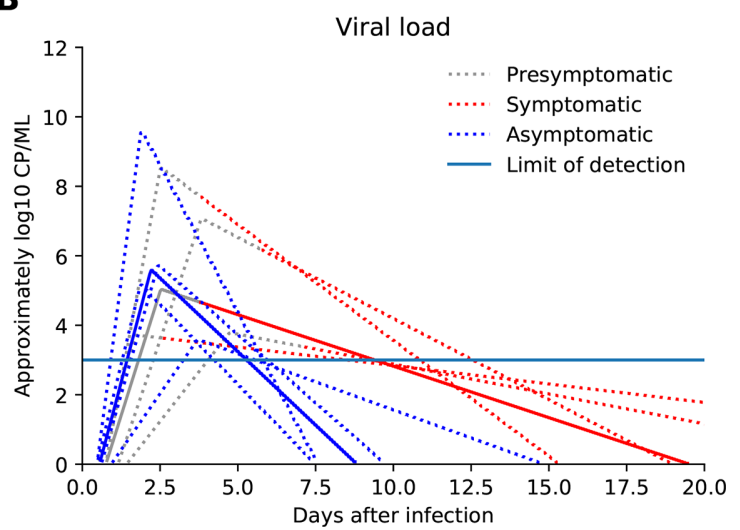

D

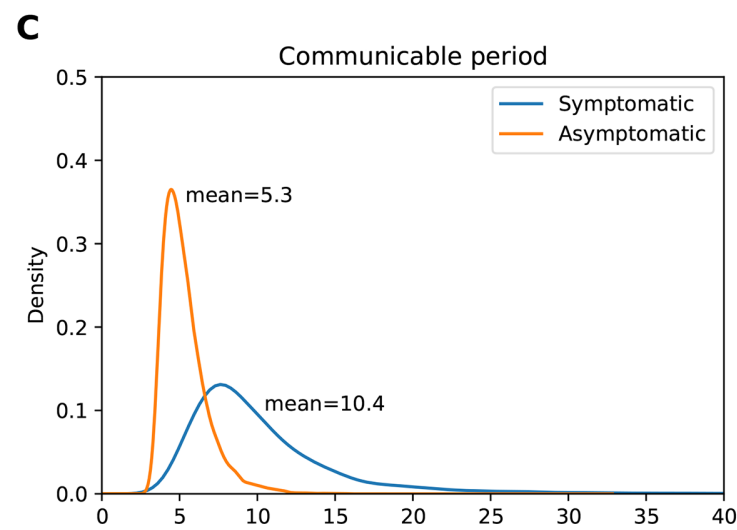

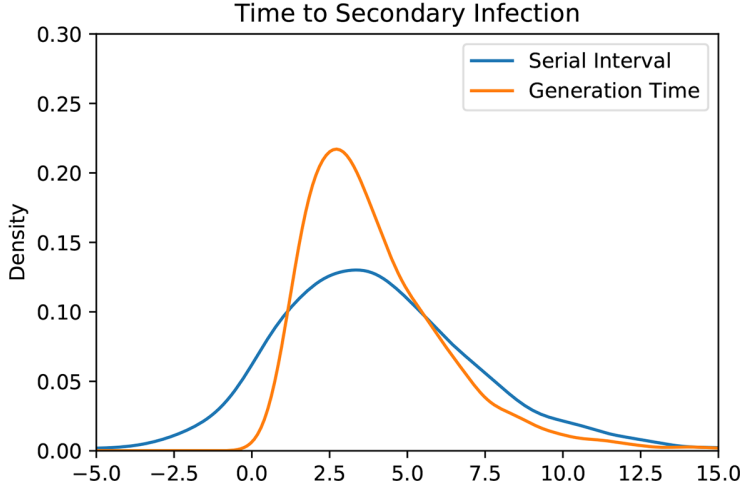

Figure 2 Transmissibility (A) and viral load models (B) for five symptomatic (red lines) and five asymptomatic individuals (blue lines). The $x$-axis represents days after infection. The $y$-axis represents the average number of infections per day over the course of infection. The red dots are infection events. The curves of viral load of these individuals follow the timeline of transmissibility, but with slower rate of decline, and viral load roughly following $\log _{10}$ copies $/ \mathrm{ml}$ of virus. The horizontal line reflects the limit of detection of a test under which the sensitivity of test decreases proportionally to viral load. (C) Density functions of communicable period for symptomatic and asymptomatic carriers. (D). Density functions of serial interval and generation time for symptomatic carriers. All densities were estimated from 10000 replicate simulations.

tract (URT) and 15 days for lower respiratory tract, with a maximum duration of RNA shedding of 83 and 59 days, respectively. However, the probability of detecting infectious the virus drops below $5 \%$ after 15.2 days postonset of symptoms. ${ }^{14}$

While symptomatic carriers have much higher transmissibility probabilities compared with asymptomatic carriers, recent studies found little to no difference in viral load between presymptomatic, asymptomatic and symptomatic persons. ${ }^{18-22}$ However, asymptomatic carriers could have faster clearance. ${ }^{1623}$ For example, Yang et al ${ }^{17}$ reported a median duration of viral shedding of 8 days (IQR : 2-12) for asymptomatic and 19 days (IQR: 16-24) for symptomatic carriers. We assumed that the lower overall infectivity of asymptomatic carriers compared with symptomatic cases with similar initial viral loads was due to different immune responses to the infection. One hypothesis is that asymptomatic carriers have reduced viral transmission due to the lack of viral expectoration via cough and sneezing. ${ }^{24}$ The immune system of asymptomatic carriers could potentially fight the virus more efficiently and clear it faster.
Similar to a study by Larremore et al, ${ }^{25}$ we modelled individual viral load patterns as piecewise functions that follow individual transmissibility curves. We extended the tails of the distributions to reflect a longer viral shedding period than communicable period and adjusted the distribution intensities, so that viral loads are proportional to the biological-driven portion of the transmissibility probabilities, and symptomatic carriers and asymptomatic carriers had similar initial viral loads (figure 2B).

\section{Sensitivity of tests}

We model the sensitivity of tests as both the LOD and clinical sensitivity. LOD is affected by the number of virions obtained from infected individuals based on their stage of infection. Clinical sensitivity can be affected by clinically relevant real-life situations (eg, variations among specimen sources, the timing of sampling and the experience of medical staff). We modelled LOD as a cut-off value where the test's sensitivity decreases proportionally to viral load when viral load is lower than the cut-off value but stays constant when the viral load is above the cut-off value (figure $2 \mathrm{~B}$ ). 
Patient and public involvement

No patient involved.

\section{RESULTS}

\section{Model validation}

We implemented and tested multiple strategies to model SARS-CoV-2 transmissibility, including using normal distributions, for both symptomatic and asymptomatic carriers. ${ }^{11}$ We validated our models extensively using simulations that summarised the final outputs of the models, including generation time; serial intervals and proportions of asymptomatic, presymptomatic and symptomatic infections. We generated secondary infection events using a transmissibility density function, which is affected by individual reproduction number, incubation period $^{2}$ and communicable period. The average number of infection events agreed well with specified reproduction numbers. The average communicable period was 10.30 days for symptomatic cases and 5.35 for asymptomatic carriers (figure 2C). ${ }^{16}{ }^{26}$ Due to the long tails of the distributions used, some infected individuals had extralong communicable periods lasting more than 20 days, which corresponds with observed outlier SARS-CoV-2 cases. ${ }^{26}$

The distribution of generation time, defined as the time between the infection in the source and infection in the recipient for source-recipient transmission pairs, was $4.25 \pm 2.75$ days $($ mean $\pm \mathrm{SD})$ days. This result is more spread out, and, therefore, more conservative, than reported in Ferretti et al (5.0 \pm 1.9 days; figure 2D). ${ }^{11}$ The distribution of serial intervals, defined as days between the primary case developing symptoms and secondary case developing symptoms, from 10000 infection events, followed a normal distribution with a mean of 4.25 and SD of 3.62. This result agrees with estimated mean serial intervals of $4.6^{27}$ and 3.96 days. ${ }^{28}$ The serial intervals followed a normal distribution with approximately $12 \%$ of pairs showing negative serial intervals, that is, the infected individuals showing symptoms before the infector (figure 2D). This is due to the wide spread of presymptomatic transmission times and is consistent with observed data. ${ }^{27}$ The proportions of asymptomatic, presymptomatic and symptomatic transmissions were $6 \%$, $48 \%$ and $44 \%$, respectively, with the default parameters we used. ${ }^{12}$

\section{Impact of testing frequency}

We assumed (1) use of an RT-PCR test with a clinical sensitivity of 0.95 and 1-day turnaround time and (2) a worstcase scenario for which everyone studied was infected just prior to quarantine. We quarantined people from 1 to 14 days and tested individuals at the end of the quarantine period. Note, we defined the day of testing as the day on which test results were obtained. In practice, an RT-PCR test conducted on 'day 11' would be administered at the beginning of day 10 and the result received at the beginning of day 11, whereas a rapid antigen test conducted on 'day 11' would be administered at the beginning of day 11 with results available shortly thereafter.

Our simulations found testing significantly reduced the PQTR in the subsequent work cohorts of quarantined individuals (figure 3A). The PQTR for 14-day quarantine was reduced from $0.12 \%$ to $0.006 \%$ when people were tested before releasing them from quarantine. Using a 95\% sensitivity RT-PCR test, a 9-day quarantine with a prerelease test resulted in a PQTR of $0.09 \%$. This risk is slightly lower than what is expected with a test-free 14-day quarantine. A longer quarantine would be needed if using a less-sensitive test. For example, a test with $80 \%$ sensitivity would require an 11-day quarantine to achieve similar risk mitigation. Notably, testing at the beginning of the quarantine, such as for a 1-day quarantine, had no effect because we assumed all individuals were infected recently and had no detectable viral load at the beginning of the simulation.

Increasing the number of tests led to shorter quarantines outperforming a test-free 14-day quarantine, mostly by reducing false-negatives through repeated testing. Assuming a 95\% sensitivity RT-PCR test and 1-day turnaround time, the 'quickest' way to release people from quarantine was a 6-day quarantine with tests on days 4, 5 and 6 . Individuals would be released at the end of day 6 if all three tests were negative. The next best option was a 7-day quarantine with tests on days 4, 5 or 6 and 7 (table 2). Adding more quarantine days or more tests would improve the performance of these strategies but would incur unnecessary costs and burden to those under quarantine.

\section{Impact of test sensitivity}

Tests that were less sensitive increased the chance of false negatives, which resulted in higher PQTR (figure 3A,B). Based on simulations of a 10-day quarantine with an RT-PCR test performed 1 day before release, a test with 90\% sensitivity would perform comparably to a 9-day quarantine with a test with $95 \%$ sensitivity. RT-PCR has a low LOD and can detect the RNA of SARS-CoV-2 from samples with $10^{3}$ copies $/ \mathrm{mL}^{30}$ to as little as $10^{2}$ copies/ mL. ${ }^{31}$ Antigen tests have a higher LOD of $10^{5}-10^{6}$ copies $/ \mathrm{mL}^{32}$ and would have a lower chance of detecting COVID-19 carriers at the beginning and end of infection. Based on the false-negative rate, the LOD of tests had a substantial impact on clinical sensitivity at the beginning of infection, which reduced around days 5 and 6 when carriers have the highest viral load and increased at a later stage of infection (figure 3C). As expected, tests with higher LOD have lower clinical sensitivities, therefore higher PQTR (figure 3D).

\section{Impact of the proportion of asymptomatic carriers}

Our quarantine strategy is more effective for asymptomatic than symptomatic carriers because asymptomatic carriers have a shorter communicable period and are less likely to infect others if not detected and released from quarantine. Therefore, the quarantine would be more effective if there are a higher percentage of asymptomatic carriers in the population. The PQTR of all quarantine 

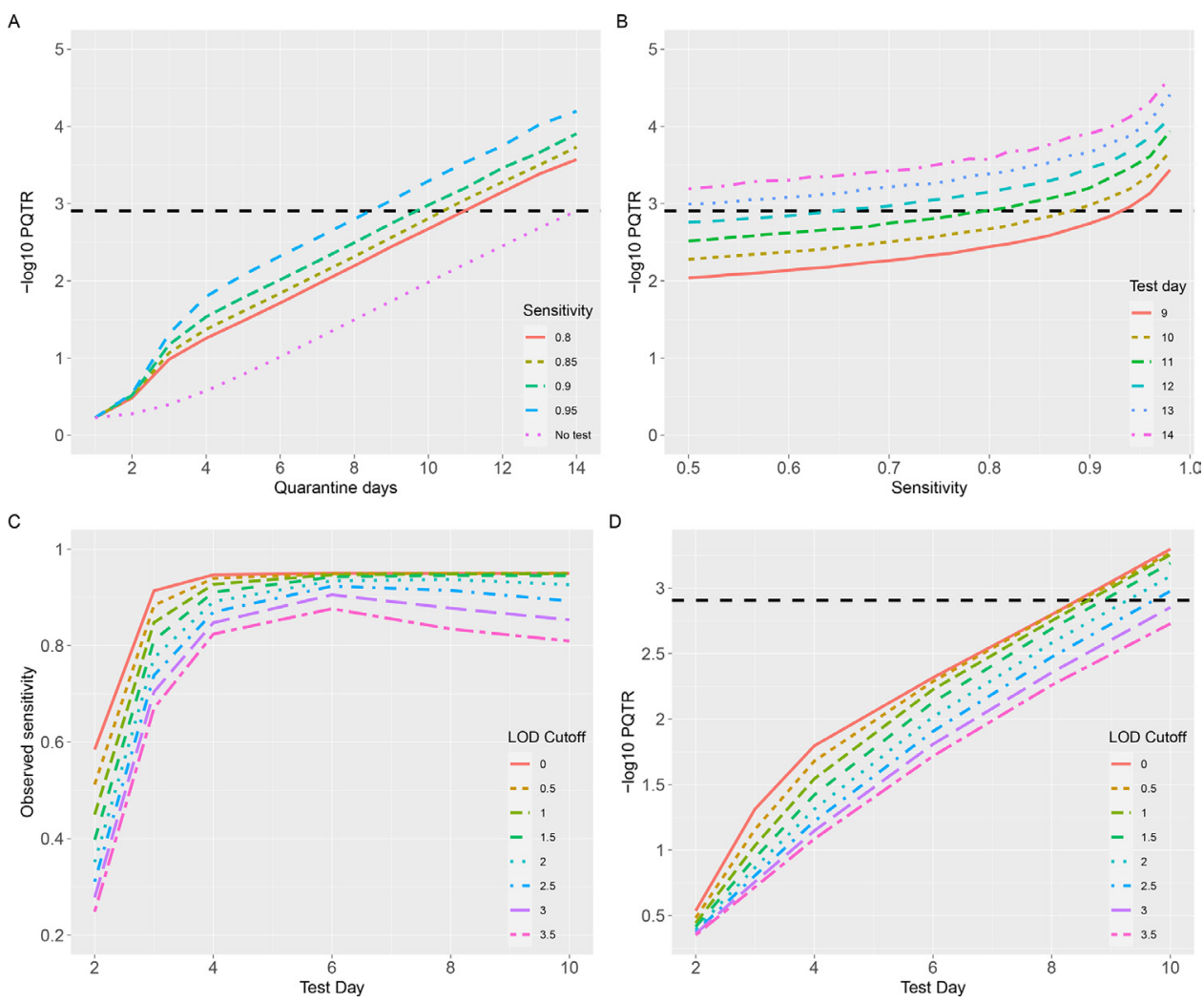

Figure 3 (A) PQTR for quarantine from 1 to 14 days with and without testing on the day before release. (B) Impact of test sensitivity on PQTR. The results are based on a 10-day quarantine with testing on the day before release. (C) The observed sensitivity, derived from the proportion of false-negatives caused by the low viral load of carriers, of tests with a baseline sensitivity of $95 \%$ when performed at days $2,3,4,6$, 8, and 10 of quarantine. (D) PQTR of the quarantine strategies with the same tests as conducted in. (C) also performed at the end of quarantine with a 1 day turnaround time. PQTR, postquarantine transmission risk.

strategies were higher in populations with a higher proportion of symptomatic cases. However, the relative performances of quarantine strategies were not affected by the proportion of asymptomatic cases.

\section{Impact of physical distancing}

Reproduction numbers measure the ability of the disease to infect others. The effective transmissibility, $\mathrm{R}_{\mathrm{e}}$ indicates the actual transmissibility accounting for behaviours, such as mask use or social distancing. In our model, real-time $\mathbf{R}_{e}$ is zero during quarantine and positive based on working environments after release. We used distancing factors to model social distancing in the working environments, which affect the probabilities that carriers infect others, but not their viral loads and probabilities to be detected.

As shown in online supplemental table 1, the PQTR of both test-free and test-assisted quarantine strategies was higher if released individuals work in an enclosed environment without social distancing than in a more

Table 2 Parameters used by six simulated SARS-CoV-2 tests

\begin{tabular}{llllll} 
Test name & Peak sensitivity & Limit of detection & Turnaround time & Average sensitivity & Average sensitivity on optimal test days \\
\hline PCR95 & $95 \%$ & 1 & 1 day & $91 \%$ & $94 \%$ \\
PCR90 & $95 \%$ & 2 & 1 day & $86 \%$ & $92 \%$ \\
PCR80 & $85 \%$ & 2 & 1 day & $77 \%$ & $83 \%$ \\
PCR70 & $80 \%$ & 3 & 1 day & $66 \%$ & $75 \%$ \\
Ag80 & $95 \%$ & 3 & 1 hour & $79 \%$ & $86 \%$ \\
Ag65 & $80 \%$ & 4 & 1 hour & $59 \%$ & $66 \%$ \\
\hline
\end{tabular}

Parameters used by six simulated SARS-CoV-2 tests. Peak sensitivity refers to sensitivity caused by factors not related to test themselves. Limit of detection refers to cut-off values for viral load below which tests have lower sensitivity proportional to viral load. Turnaround time refers to time after which test results can be obtained. Average sensitivity is the averaged observed sensitivities if tests are performed during the communicable periods (with positive viral loads) for 10000 random simulated individuals with default parameters who were infected at time 0 . Average sensitivity on optimal test days is the average observed sensitivity for quarantine strategies that test individuals at selected days that result in lowest PQTR.

PQTR, postquarantine transmission risk. 
protected environment with social distancing and mask use. However, compared with a test-free quarantine, in which all asymptomatic carriers are released and become more infectious in environments with less distancing, test-assisted quarantine strategies detect most carriers and release them only after they are no longer infectious. The PQTR of test-assisted quarantines increases slower than test-free quarantines when simulating scenarios with progressively decreasing social distancing practices. Most of the test-assisted quarantine strategies (with shorter quarantine intervals) outperformed the test-free quarantines (online supplemental table 1). Therefore, test-assisted strategies are more effective than test-free quarantines for the assembling of individuals without social distancing.

\section{Impact of individual viral loads}

In contrast to physical distancing that affects only postquarantine transmission probabilities, individual viral loads impact both the probability that a carrier is detected during quarantine, and their transmission probability after the quarantine period. Using a strategy that tests individuals 24 hours before the end of a 10-day quarantine, we simulated five groups of individuals with varying overall viral loads (from half to two times) and estimated observed test sensitivities and PQTR for four tests with varying sensitivity and limits of detection. Because test sensitivities plateau when viral loads exceed the LOD of tests, the impact of viral loads on postquarantine transmission probabilities exceeds its impact on test sensitivities, leading to roughly linear increase in PQTR with increased viral loads. This trend is appreciable in figure $4 \mathrm{~A}$.

\section{Impact of incubation periods}

The length of the SARS-CoV-2 incubation period, or the time from exposure to the development of symptoms, determines the likelihood that the carrier shows symptoms during a quarantine intervention. Incubation period length further influences the viral load and communicable period of a carrier, and, therefore, their postquarantine transmission probabilities. In figure 4B, we present our results of varying the relative incubation length of viral infection from half to two times in duration. It can be appreciated that PQTR increases exponentially, independent of testing strategy, as the incubation period lengthens.

\section{Optimal strategies for widely used SARS-CoV-2 tests}

PCR-based tests can detect the existence of SARS-CoV-2 RNA from URT samples of recovered patients for up to 12 weeks, even when the replication-competent virus was not detected more than 3 weeks after symptom onset. $^{33} 34$ The clinical sensitivity of SARS-CoV-2 tests is affected by test design, the viral loads of infected individuals and many human and nonhuman factors. Therefore, although sensitivity for COVID-19 tests that are derived from testing samples with the SARS-CoV-2 virus in a lab environment is generally very high (usually $>95 \%$ ), the real-world performance of these tests can be significantly poorer. ${ }^{35}$ Reported clinical sensitivities include $71 \%$, $86 \%,{ }^{37} 82 \%$ in a more recent retrospective study, ${ }^{38}$ and a systematic review of 34 studies involving 12057 patients showed test sensitivities ranging from $42 \%$ to $98 \%$ with a median of $89 \% .{ }^{39}$ In addition to cross-study differences, another meta-analysis of 1330 samples from seven previously published studies showed median sensitivities that start from $0 \%$ at the onset of infection, $34 \%$ at day 4 , peak at $80 \% 1$ day after the onset of symptoms and decrease to $34 \%$ at day $21 .^{40}$ More recent studies, however, attributed the lower sensitivity of the earlier studies partly to the use of oropharyngeal instead of nasopharyngeal swabs and found that RT-PCR tests generally have more than $90 \%$ sensitivity. ${ }^{41}$

Test-assisted quarantine strategies offset the increased PQTR caused by shorter quarantine days through the identification of virus carriers before they develop
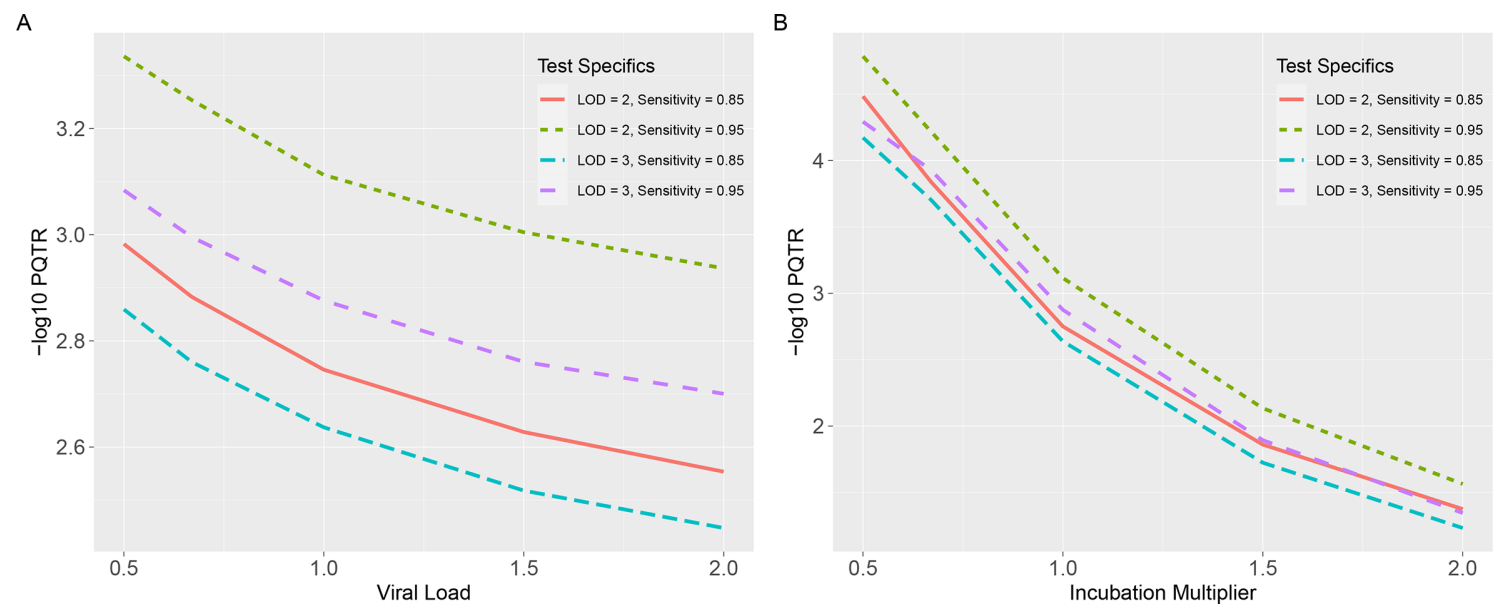

Figure 4 (A) PQTR for a 10-day quarantine strategy with four different tests administered on the day before release, for people with viral load that are $1 / 2,2 / 3,1,1.5$ and 2.0 times of the average viral load of the simulated population. (B) PQTR for the same quarantine strategies and tests, for people with 1/2, 2/3, 1, 1.5 and 2.0 times of the average incubation period of the simulated population. PQTR, postquarantine transmission risk. 
symptoms. Effective test-assisted quarantine strategies test individuals at the peak of their viral loads at which tests are most sensitive and use repeated testing to further reduce false-negatives and increase sensitivity. To evaluate the performance of commonly used SARS-CoV-2 tests, we modelled six SARS-CoV-2 tests that include (a) a bestcase scenario in which high-sensitivity RT-PCR tests are applied to samples collected from nasopharyngeal or midturbinate swabs, (b) a common scenario with a regular RT-PCR test on anterior nasal swabs that misses positives due to lower viral loads (Cycle threshold >30-32), (c) two RT-PCR tests with lower sensitivities due to poor quality of tests, protocol or administration of tests and (d) two antigen tests with lower sensitivity, but faster turnaround time (1 hour) and lower cost (table 2$)$. Due to variations of viral loads at different stages of infection, ${ }^{40}$ we model the sensitivities of the tests with a baseline peak sensitivity value that reflects common factors unrelated to the test themselves and LOD cut-off values that reflect analytic sensitivities of the tests. The observed sensitivity of these tests varies over the course of the infection (figure 3C). The average sensitivities, if the tests are applied everyday during the communicable periods with positive viral loads, range from $66 \%$ to $91 \%$ for four RT-PCR tests and $59 \%$ to $79 \%$ for two antigen tests.

Assuming default parameters for $\mathrm{R}_{0}$, no social distancing after quarantine and proportion of asymptomatic carriers in the population, we evaluated the performance of quarantine strategies with all possible combinations of testing schedules using these six tests for those undergoing quarantine with either simultaneous or mixed onset of infections (tables 3 and 4 and online supplemental table 1). We found numerous quarantine strategies that performed better than a test-free 14-day quarantine and reported only those with the shortest quarantine or smallest number of tests. Our results indicate that quarantine on people with mixed onset of infection has much lower PQTR (generally $1 / 10$ ) and a wider choice of safe test-assisted quarantine strategies compared with quarantine with the simultaneous onset of infection (online supplemental table 1).

Considering only strategies that outperform a standard 14-day quarantine for individuals with both simultaneous and mixed onset of infections, a single RT-PCR test administered 1 or 2 days before the end of quarantine could reduce the duration of quarantine to 10 days. Two RT-PCR tests administered on days 6 or 7 and then day 8 could reduce the duration to 8 days. In cases where the shortest quarantine is needed, a 6-day quarantine with tests on days 4,5 and 6 using a highly sensitive RT-PCR test can be justified. Longer quarantine times are required for tests with lower sensitivity. For example, two more days of quarantine would be needed if the test PCR70 is used (tables 2 and 3 ).

Due to their high LOD, antigen tests are less sensitive in detecting the presence of the SARS-CoV-2 virus at early and later stages of infections. A single antigen test with test administered on day 9 or 10 could reduce the quarantine to 11 days. Persons in quarantine who tested negative at the beginning of days 8 and 9 could be released at the beginning of day 10 if they do not show any symptoms (a 9-day quarantine with tests on days 7 and 8). Longer quarantines would be needed for antigen tests with lower sensitivity. Shorter quarantines, even with daily antigen testing, did not achieve the same level of safety as a testfree 14-day quarantine.

For applications in which higher residual risks could be tolerated, or be mitigated by, for example, strict physical distancing and postquarantine symptom monitoring, a 10-day test-free quarantine with a PQTR of $1.05 \%$ is recommended by the December 2020 guideline from the US CDC. A number of test-assisted quarantines, including 6-day quarantine with one RT-PCR test and 5-day quarantine with two RT-PCR tests, provide lower PQTR than 10-day test-free quarantine, which was suggested by the US CDC. Quarantine strategies with testing at the beginning and end of quarantine incur residual risk. As a real-world example, the New York travel recommendation resembles an 8 day quarantine strategy with testing on days 1 and 8 , The PQTR of this strategy is $0.024 \%$, which is $60 \%$ higher than a 14-day quarantine even if we assume that travelling adds no additional risk of infection. We report in table 4 those strategies with either the shortest quarantine time or smallest number of tests.

\section{DISCUSSION}

Using our flexible and publicly available COVID-19 Python-coded simulator (https://ictr.github.io/covid19outbreak-simulator/), we modelled the efficacy of foreshortened quarantine strategies with and without the concurrent implementation of COVID-19 testing. We evaluated the efficacy of these test-assisted quarantines through modulating the simulated test sensitivities and turnaround times of carriers at different stages of infection. Furthermore, we modelled the effect of specific implementation scenarios, based on our existing knowledge on the progression of SARS-CoV-2 infection and performance of common RT-PCR and antigen tests. We hypothesised that COVID-19 testing would allow for a shorter quarantine than a test-free 14 or 10-day quarantine strategy. The simulations identified specific shortened quarantined strategies that could be effective.

Using a relatively sensitive RT-PCR test and current lab protocols, ending a quarantine at day 10 with a single RT-PCR test performed 1 or 2 days before release outperformed a test-free 14-day quarantine for both simultaneous and mixed-onset COVID-19 exposures (table 3). Exceptionally short quarantines, including a 6-day quarantine, were justifiable if repeated high-sensitivity RT-PCR testing was performed on days 4,5 and 6 , and even shorter quarantine strategies could be used for applications with higher tolerance to PQTR (table 4). Quarantining individuals with mixed exposure instances yielded a wider range of effective quarantine strategies than did a cohort of simultaneously exposed individuals. Epidemiologically, 
Table 3 Optimal quarantine strategies with the shortest duration or fewest numbers of tests that had better performance than a 14-day quarantine.

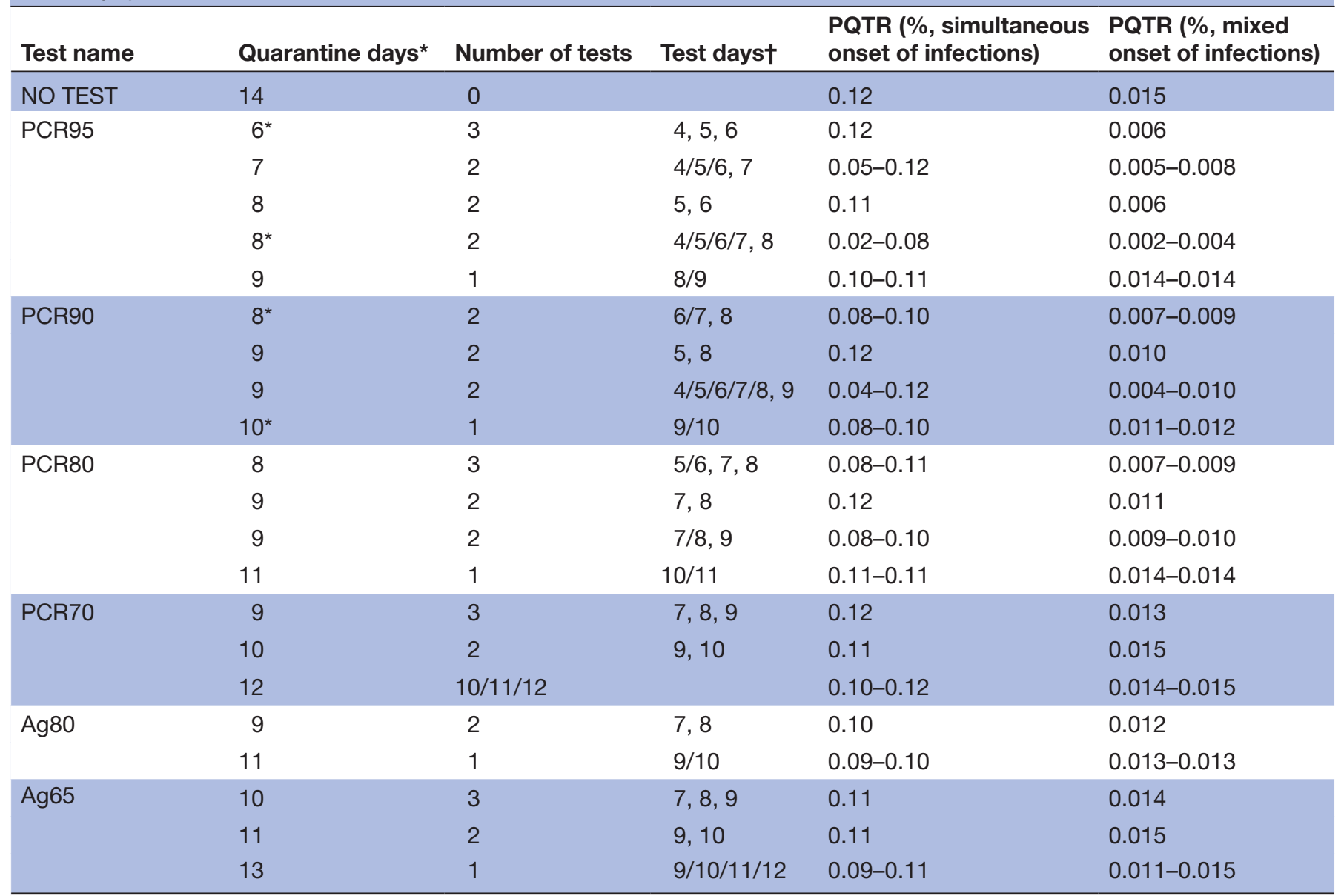

Quarantine strategies with the shortest duration or fewest numbers of tests that had better performance than a 14-day quarantine, for four RT-PCR tests with a 1-day turnaround time, and two antigen tests with 1-hour hour turnaround time. Details of these tests are listed in Table 2. Test days represent days at which test results would be available at the end of day. PQTR is calculated for (1) simultaneous onset of infections when infection happens simultaneously from a single source of contact or event and (2) mixed onset of infections when onsets of infection are assumed to occur any time during the course of infection for asymptomatic carriers, and any time during incubation period for symptomatic cases. Only quarantine strategies with lower PQTR than those of a 14-day quarantine in both cases are listed. Strategies with more tests or longer quarantine time than the listed strategies will perform better and are not listed. A complete list of quarantine strategies is provided in online supplemental table 1.

*Strategies that are recommended are marked by.

†Strategies with the same last test day and for which various different earlier test days gave similar results are combined with the other test days separated by ' $/$ '. The test days are days on which the tests are administered. The PCR tests are assumed to be administered at the beginning of the day and the Antigen tests are assumed to be administered at the end of the day.

PQTR, postquarantine transmission risk; RT-PCR, reverse transcription-PCR.

we found the average communicable period for persons with symptomatic cases of COVID-19 was 10.30 days compared with 5.35 for asymptomatic carriers.

We estimated PQTR of test-assisted quarantined based on a mixed population with varying properties such as viral load, incubation period, communicable period, proportion of asymptomatic carriers. However, age $e^{4243}$ male gender, ${ }^{44}$ elevated risk profile ${ }^{45}$ are all associated with the poor prognosis of COVID-19 diseases ${ }^{46}$ and mortality, ${ }^{47}$ so different quarantine strategies might be needed for cohorts consisting with seniors, men or people with multiple complications.

The false-positive rates of RT-qPCR tests are associated with patient age, sex and time since diagnosis, although the association is best explained by individual viral loads. ${ }^{48}$ Since the average differences in viral loads and resulting differences in false-negative rates and PQTR between men and women are relatively small ( $\sim 5 \%$ in false-negative rates) compared with differences between people under and over the age of $40(\sim 20 \%$ in false-negative rates $),{ }^{48}$ a more stringent test-assisted quarantine strategy should be used for cohorts with predominantly people over the age of 40. Individuals with multiple comorbidities tend to have shorter incubation periods, higher viral loads and worse prognoses. We found each of these factors increases the efficiency of test-assisted quarantines (as shown in figure 4); therefore, we suggest that our recommended quarantine strategies should be applicable to cohorts with elevated risk profiles. 
Table 4 Optimal quarantine strategies with the shortest duration or fewest numbers of tests that had better performance than a 10-day quarantine

\begin{tabular}{llllll}
\hline Test name & Quarantine days ${ }^{*}$ & Number of tests & Test days & $\begin{array}{l}\text { PQTR (\%, simultaneous } \\
\text { onset of infections) }\end{array}$ & $\begin{array}{l}\text { PQTR (\%, mixed } \\
\text { onset of infections) }\end{array}$ \\
\hline NO TEST & 10 & 0 & & 1.01 & 0.154 \\
PCR95 & $5^{*}$ & 2 & $3 / 4,5$ & $0.47-0.75$ & $0.028-0.047$ \\
PCR90 & 6 & 1 & $5 / 6$ & $0.60-0.87$ & $0.097-0.110$ \\
PCR80 & $6^{*}$ & 1 & 6 & 0.96 & 0.110 \\
\hline PCR70 & 6 & 2 & $4 / 5.6$ & $0.80-0.84$ & $0.078-0.107$ \\
& 8 & 1 & $6 / 7 / 8$ & $0.57-0.83$ & $0.085-0.105$ \\
Ag80 & 7 & 2 & $5 / 6,7$ & $0.69-0.89$ & $0.091-0.108$ \\
& $6^{*}$ & 1 & 8 & 0.74 & 0.134 \\
Ag65 & $7^{*}$ & 2 & 4,5 & 0.94 & 0.089 \\
& 7 & 1 & 6 & 0.81 & 0.117 \\
& 8 & 3 & $3 / 4,5,6$ & $0.80-0.93$ & $0.094-0.109$ \\
& 8 & 2 & $4 / 5,6$ & $0.80-0.96$ & $0.103-0.123$ \\
\hline
\end{tabular}

Quarantine strategies with the shortest duration or fewest numbers of tests that had better performance than a 10-day quarantine, for four RT-PCR tests with a 1-day day turnaround time and two Antigen tests with a 1-hour hour turnaround time. Details of these tests are listed in Table 2.

PQTR, postquarantine transmission risk; RT-PCR, reverse transcription-PCR.

The emergence and wide spread of new variants of the SARS-CoV-2 virus pose new challenges to effective application of quarantine strategies. For example, the $614 \mathrm{G}$ variants propagate more quickly than the wide type ${ }^{49}$ and preliminary studies suggest that the 501Y.V2 variant is associated with a higher viral load. The variants might also evade detection by specific viral diagnostic tests although most commercial RT-PCR tests have multiple targets to detect the virus and should continue to work. Although there is insufficient data for us to develop variant-specific quarantine strategies, we recommend the use of more stringent quarantine strategies in areas with high prevalence of these new variants.

Strengths of our approach include repeated and systematic simulation comparisons that allow us to confidently draw conclusions regarding the efficacy of these quarantine models based on our accurate probabilistic modelling of person-to-person COVID-19 spread in well-mixed populations. Strengths of our model include effective implementation and accurate modelling of COVID-19 spread in diverse real-world scenarios. ${ }^{7}$ A potential limitation of our modelling technique is our use of probabilistic modelling. We simulated the occurrence of infection and recovery events at random based off measured parameter probabilities of COVID-19 spread within a network or between networks. This limits the contribution of geographical constraints or nonrandom social interaction. Furthermore, we found lengthened incubation periods diminishes the efficacy of quarantine strategies. Therefore, it is critically important to understand variations in incubation period, especially for test-assisted quarantine strategies with shorter quarantine durations. Since individuals with shorter incubation periods are more likely to show symptoms during quarantine (figure $4 \mathrm{~b}$ ), individuals at the tail of the distribution of incubation period contribute to the majority of cases who show symptoms and infect others after quarantine and to the PQTR of our presented quarantine strategies.

The literature on foreshortened and test-assisted quarantine strategies is sparse. Only two investigations similar to ours have been conducted, and currently, neither have been peer-reviewed. The first study extrapolated epidemiological parameters of COVID-19 from a real-world data set from COVID-19 testing of offshore oil rig employees. ${ }^{50}$ The second used a model to capture transmission associated with air travel and develop quality strategies to mitigate the entry of COVID-19 into new regions. ${ }^{51}$ Both studies had limitations similar to ours. They had to estimate epidemiological COVID-19 parameters from limited real-world data sets, and they had to create probabilistic models to test the efficacy of COVID-19 testing in their respective scenarios. Our base model has a distinct advantage due to its generalisability, unique flexibility and easy programming with command line prompts to simulate a wide range of scenarios. We have also iteratively tested the efficacy of the quarantine strategies in a broad range of scenarios. Collectively, these model features ensure our simulations are robust.

A pressing need exists for evidence-based COVID-19 mitigation strategies. Currently, deviations from a 14-day postexposure quarantine have little substantiating evidence supporting their utility. Despite a lack 
of systematic simulation modelling, shortened and testassisted quarantine strategies are being implemented out of logistical necessity. Our work fills a clear gap in our understanding of quarantine strategy efficacy by comprehensively simulating alternative isolation strategies. The increased efficacy we identified using test-assisted quarantine strategies should also be appreciated beyond their ability to shorten the overall quarantine. A major difference between test-free and test-assisted quarantine is that test-assisted quarantines identify asymptomatic carriers and carriers who have recovered but are still shedding, that is, those who would pass test-free quarantine unnoticed. Therefore, the 10-day test-assisted quarantine we identified or the even shorter quarantines with repeated RT-PCR testing demonstrate superior efficacy at mitigating viral spread then a test-free quarantine process. This underscores the value of test-assisted quarantine as it reduces the probability of subsequent quarantine failure while also releasing uninfected individuals from quarantine earlier.

We comment that this recommendation is in line with the recent recommendations from the CDC. For example, the CDC recommended 10-day and 7-day quarantines with an RT-PCR test performed within 48 hours before the end of the quarantine, which are similar to our recommended 9-day and 6-day quarantines with tests performed within 24 hours before the end of the quarantine. Due to the wide range of test sensitivities, the CDC reported wide ranges of PQTR and recommended, among other methods, continued monitoring of symptoms after releasing from quarantine to mitigate the risk caused by the use of tests with lower sensitivities.

Test-assisted quarantines require the administration of SARS-CoV-2 tests. The costs of testing depend on the size of cohort, number and type of tests and could outweigh the benefits of reduced quarantine time. We recommend optimal test-assisted quarantine strategies for different types of tests with one or more tests during quarantine and allow the use of most cost-effective strategies for particular applications. Notably, testing cohorts with uninfected individuals, especially repeated testing of large cohorts, generates false positives. RT-PCR tests have very high specificity (between $99.9 \%$ and $99.95 \%$ ) and produce false positives only through human errors during preanalytical and analytical processes. These values are higher than those of lower specificity antigen tests (eg, 98.5\% for BinaxNow). Therefore, the F.D.A. recommends positive tests obtained by antigen tests are confirmed with an RT-PCR test. Unless a physician suggests otherwise, people who test positive should complete a 10-day isolation as recommended by the CDC.

Effective vaccines can generate host immunity to the novel SARS-CoV2 virus. A clear direction for future research involves modelling viral spread in populations with varying degrees of vaccine-induced immunity. Specifically, questions that remain unaddressed include the efficacy of the quarantine strategies we present given known vaccination status, the timing of recent vaccination and the vaccination status of the population or subpopulations of interest.

\section{CONCLUSION}

Our findings indicate that the current 14-day quarantine approach is excessively conservative, and the duration of quarantines could be significantly reduced if testing is performed during the quarantine period (tables 3 and 4). To achieve a PQTR of $0.1 \%$, comparable to a 14-day test-free quarantine, one RT-PCR test or two antigen tests could reduce the quarantine time to 10 days. More tests can further reduce the length of duration, and a 6-day quarantine can be justified with three highly sensitive RT-PCR tests. Even shorter quarantine strategies could be employed if a higher PQTR of $1 \%$, comparable to a 10-day test-free quarantine, is tolerated. We modelled uninterrupted quarantine in isolation with tests with relatively short turnaround time. If, in practice, the turnaround time of a test is longer than 1 day, the quarantine should be extended to wait for the results of the tests.

The effectiveness of test-assisted quarantine depends on the sensitivity of tests. Because SARS-CoV-2 tests have lower sensitivity when administered at an early stage of infection with low viral loads, quarantines shorter than 6 days will be ineffective even with daily SARS-CoV-2 testing. Quarantine strategies with testing at the beginning and end of quarantine or travelling with pretravel testing could incur residual risk. Tests with lower than $85 \%$ clinical sensitivity are unlikely to reduce the length of quarantine, and we do not recommend using testassisted quarantine with novel tests with unknown clinical sensitivities.

Acknowledgements The authors appreciate a scientific editor, Kat H. Sippel, $\mathrm{PhD}$, E.L.S. for her assistance in the preparation of this manuscript. Dr. Amos is a Research Scholar of the Cancer Prevention Research Institute of Texas and supported by RR170048 and National Institutes of Health Award Number 10T2HL158258-01. Rowland Pettit is a fellow under the Training in Precision Environmental Health Sciences (TPEHS) Program (N.I.H. Grant No. T32ES027801). The authors would like to thank BRASS: Baylor Research Advocates for Student Scientists for their support of Rowland Pettit.

Contributors BP designed the study, implemented the simulations and drafted the manuscript. WZ executed simulations and analysed results. BP, WZ and PY verified the data and results. PY, PGM, ALG and JM provided real-world data, industrial practices and government regulations for COVID-19 quarantines. CA supervised the study. PY, RWP and CA contributed substantially to the manuscript. All authors reviewed and agreed with the content of the manuscript.

Funding Dr. Amos is a research scholar of the Cancer Prevention Institute of Texas and supported by RR170048. He is also supported by NHLBI grant 10T2HL15825801. Mr. Pettite is supported by training grant T32ES027801.

Competing interests JM is an employee of International S.0.S. BP and PM are consultants to International S.0.S.

Patient consent for publication Not required.

Provenance and peer review Not commissioned; externally peer reviewed.

Data availability statement Data are available in a public, open access repository. Extra data can be accessed via the Dryad data repository at http://datadryad.org/ with the doi: 10.5061/dryad.ksn02v749.

Supplemental material This content has been supplied by the author(s). It has not been vetted by BMJ Publishing Group Limited (BMJ) and may not have been peer-reviewed. Any opinions or recommendations discussed are solely those 
of the author(s) and are not endorsed by BMJ. BMJ disclaims all liability and responsibility arising from any reliance placed on the content. Where the content includes any translated material, BMJ does not warrant the accuracy and reliability of the translations (including but not limited to local regulations, clinical guidelines, terminology, drug names and drug dosages), and is not responsible for any error and/or omissions arising from translation and adaptation or otherwise.

Open access This is an open access article distributed in accordance with the Creative Commons Attribution Non Commercial (CC BY-NC 4.0) license, which permits others to distribute, remix, adapt, build upon this work non-commercially, and license their derivative works on different terms, provided the original work is properly cited, appropriate credit is given, any changes made indicated, and the use is non-commercial. See: http://creativecommons.org/licenses/by-nc/4.0/.

\section{ORCID iD}

Christopher I Amos http://orcid.org/0000-0002-8540-7023

\section{REFERENCES}

1 Lee VJ, Chiew CJ, Khong WX. Interrupting transmission of COVID-19: lessons from containment efforts in Singapore. J Travel Med 2020;27.

2 Lauer SA, Grantz KH, Bi Q, et al. The incubation period of coronavirus disease 2019 (COVID-19) from publicly reported confirmed cases: estimation and application. Ann Intern Med 2020;172:577-82.

3 Brooks SK, Webster RK, Smith LE, et al. The psychological impact of quarantine and how to reduce it: rapid review of the evidence. The Lancet 2020;395:912-20.

4 Johansson MA, Wolford $\mathrm{H}$, Paul P, et al. Reducing travel-related SARS-CoV-2 transmission with layered mitigation measures: symptom monitoring, quarantine, and testing. medRxiv 2020.

5 Quilty BJ, Clifford S, Flasche S, et al. Quarantine and testing strategies in contact tracing for SARS-CoV-2: a modelling study. medRxiv 2020.

6 Ashcroft P, Lehtinen S, Angst DC, et al. Quantifying the impact of quarantine duration on COVID-19 transmission. medRxiv 2020.

7 Peng B, Amos Cl. Population simulations of COVID-19 outbreaks provide tools for risk assessment and continuity planning. medRxiv 2020.

8 Wu JT, Leung K, Leung GM. Nowcasting and forecasting the potential domestic and international spread of the 2019-nCoV outbreak originating in Wuhan, China: a modelling study. Lancet 2020;395:689-97.

9 Liu Y, Gayle AA, Wilder-Smith A, et al. The reproductive number of COVID-19 is higher compared to SARS coronavirus. J Travel Med 2020;27. doi:10.1093/jtm/taaa021. [Epub ahead of print: 13 Mar 2020].

10 Tang B, Wang X, Li Q, et al. Estimation of the transmission risk of the 2019-nCoV and its implication for public health interventions. J Clin Med 2020;9:462.

11 Ferretti L, Wymant C, Kendall M, et al. Quantifying SARS-CoV-2 transmission suggests epidemic control with digital contact tracing. Science 2020;368:eabb6936.

12 Ferretti L, Wymant C, Kendall M, et al. Quantifying SARS-CoV-2 transmission suggests epidemic control with digital contact tracing. Science 2020;368. doi:10.1126/science.abb6936. [Epub ahead of print: 0805 2020]

13 Wölfel R, Corman VM, Guggemos W, et al. Virological assessment of hospitalized patients with COVID-2019. Nature 2020;581:465-9.

14 van Kampen JJA, van de Vijver D, Fraaij PLA, et al. Shedding of infectious virus in hospitalized patients with coronavirus disease-2019 (COVID-19): duration and key determinants. medRxiv 2020. doi:https://doi.org/10.1101/2020.06.08.20125310

15 Young BE, Ong SWX, Kalimuddin S, et al. Epidemiologic features and clinical course of patients infected with SARS-CoV-2 in Singapore. JAMA 2020;323:1488-94.

16 Zhang Z, Xiao T, Wang Y, et al. Early viral clearance and antibody kinetics of COVID-19 among asymptomatic carriers. medRxiv 2020. doi:10.1101/2020.04.28.20083139

17 Yang R, Gui X, Xiong Y. Comparison of clinical characteristics of patients with asymptomatic vs symptomatic coronavirus disease 2019 in Wuhan, China. JAMA Netw Open 2020;3:e2010182e:e2010182.

18 Walsh KA, Jordan K, Clyne B, et al. SARS-CoV-2 detection, viral load and infectivity over the course of an infection. J Infect 2020;81:357-71.

19 Wan R, Mao Z-Q, He L-Y, et al. Evidence from two cases of asymptomatic infection with SARS-CoV-2: are 14 days of isolation sufficient? Int J Infect Dis 2020;95:174-5.
20 Le TQM, Takemura T, Moi ML, et al. Severe acute respiratory syndrome coronavirus 2 shedding by travelers, Vietnam, 2020. Emerg Infect Dis 2020;26:1624-6.

21 Lavezzo E, Franchin E, Ciavarella C, et al. Suppression of a SARS-CoV-2 outbreak in the Italian municipality of VO'. Nature 2020;584:425-9.

22 Corcorran MA, Olin S, Rani G, et al. Prolonged persistence of PCRdetectable virus during an outbreak of SARS-CoV-2 in an inpatient geriatric psychiatry unit in King County, Washington. Am J Infect Control 2021;49:293-8.

23 Van Vinh Chau N, Lam VT, Dung NT, et al. The natural history and transmission potential of asymptomatic severe acute respiratory syndrome coronavirus 2 infection. Clin Infect Dis 2020;71:2679-87.

24 Gao M, Yang L, Chen X, et al. A study on infectivity of asymptomatic SARS-CoV-2 carriers. Respir Med 2020;169:106026.

25 Larremore DB, Wilder B, Lester $\mathrm{E}$, et al. Test sensitivity is secondary to frequency and turnaround time for COVID-19 surveillance. medRxiv . 2020. doi:10.1101/2020.06.22.20136309. [Epub ahead of print: 27 Jun 2020

$26 \mathrm{Hu} \mathrm{Z}$, Song C, Xu C, et al. Clinical characteristics of 24 asymptomatic infections with COVID-19 screened among close contacts in Nanjing, China. Sci China Life Sci 2020;63:706-11.

27 Nishiura $\mathrm{H}$, Linton NM, Akhmetzhanov AR. Serial interval of novel coronavirus (COVID-19) infections. Int J Infect Dis 2020;93:284-6.

$28 \mathrm{Du} \mathrm{Z}, \mathrm{Xu} \mathrm{X}, \mathrm{Wu} \mathrm{Y}$, et al. The serial interval of COVID-19 from publicly reported confirmed cases. medRxiv 2020 doi:10.1101/2020.02.19.20025452

29 Du Z, Xu X, Wu Y, et al. Serial interval of COVID-19 among publicly reported confirmed cases. Emerg Infect Dis 2020;26:1341-3.

30 Vogels CBF, Brito AF, Wyllie AL, et al. Analytical sensitivity and efficiency comparisons of SARS-CoV-2 RT-qPCR primer-probe sets. Nat Microbiol 2020;5:1299-305.

31 Degli-Angeli E, Dragavon J, Huang M-L, et al. Validation and verification of the Abbott RealTime SARS-CoV-2 assay analytical and clinical performance. J Clin Virol 2020;129:104474.

32 Meyerson NR, Yang Q, Clark SK, et al. A community-deployable SARS-CoV-2 screening test using RAW saliva with 45 minutes sample-to-results turnaround. medRxiv 2020.

33 Li N, Wang X, Lv T. Prolonged SARS-CoV-2 RNA shedding: not a rare phenomenon. J Med Virol 2020;92:2286-7.

34 Xiao F, Sun J, Xu Y, et al. Infectious SARS-CoV-2 in feces of patient with severe COVID-19. Emerg Infect Dis 2020;26:1920-2.

35 Yang Y, Yang M, Shen C, et al. Evaluating the accuracy of different respiratory specimens in the laboratory diagnosis and monitoring the viral shedding of 2019-nCoV infections. medRxiv 2020. doi:10.1101/2020.02.11.20021493

36 Watson J, Whiting PF, Brush JE. Interpreting a covid-19 test result. BMJ 2020;369:m1808.

37 Holborow A, Asad H, Porter L, et al. The clinical sensitivity of a single SARS-CoV-2 upper respiratory tract RT-PCR test for diagnosing COVID-19 using convalescent antibody as a comparator. Clin Med 2020;20:e209-11.

38 Williams TC, Wastnedge E, McAllister G, et al. Sensitivity of RTPCR testing of upper respiratory tract samples for SARS-CoV-2 in hospitalised patients: a retrospective cohort study. medRxiv 2020. doi:10.1101/2020.06.19.20135756

39 Arevalo-Rodriguez I, Buitrago-Garcia D, Simancas-Racines D, et al. False-Negative results of initial RT-PCR assays for COVID-19: a systematic review. PLoS One 2020;15:e0242958.

40 Kucirka LM, Lauer SA, Laeyendecker O, et al. Variation in false-negative rate of reverse transcriptase polymerase chain reaction-based SARSCoV-2 tests by time since exposure. Ann Intern Med 2020;173:262-7.

41 Long DR, Gombar S, Hogan CA, et al. Occurrence and timing of subsequent severe acute respiratory syndrome coronavirus 2 reverse-transcription polymerase chain reaction positivity among initially negative patients. Clin Infect Dis 2021;72:323-6.

42 Euser S, Aronson S, Manders I, et al. SARS-CoV-2 viral load distribution reveals that viral loads increase with age: a retrospective cross-sectional cohort study. medRxiv 2021. doi:https://doi.org/10. 1101/2021.01.15.21249691

43 Baggio S, L'Huillier AG, Yerly S, et al. SARS-CoV-2 viral load in the upper respiratory tract of children and adults with early acute COVID-19. medRxiv 2020. doi:https://doi.org/10.1101/2020.07.17. 20155333

44 Zhang J, Yu M, Tong S, et al. Predictive factors for disease progression in hospitalized patients with coronavirus disease 2019 in Wuhan, China. J Clin Virol 2020;127:104392.

45 Booth A, Reed AB, Ponzo S, et al. Population risk factors for severe disease and mortality in COVID-19: a global systematic review and meta-analysis. medRxiv 2020. doi:https://doi.org/10.1101/2020.12. 21.20248610 
46 Soria ME, Cortón M, Martínez-González B, et al. High SARS-CoV-2 viral load is associated with a worse clinical outcome of COVID-19 disease. medRxiv 2020. doi:https://doi.org/10.1101/2020.11.13. 20229666

47 Pujadas E, Chaudhry F, McBride R, et al. SARS-CoV-2 viral load predicts COVID-19 mortality. Lancet Respir Med 2020;8:e70.

48 Levine-Tiefenbrun M, Yelin I, Uriel H, et al. Association of COVID-19 RT-qPCR test false-negative rate with patient age, sex and time since diagnosis. medRxiv 2020. doi:10.1101/2020.10.30.20222935
49 Wu K, Werner AP, Moliva Jl, et al. mRNA-1273 vaccine induces neutralizing antibodies against spike mutants from global SARSCoV-2 variants. bioRxiv 2021. doi:10.1101/2021.01.25.427948. [Epub ahead of print: 25 Jan 2021].

50 Wells CR, Townsend JP, Pandey A, et al. Optimal COVID-19 quarantine and testing strategies. Nature Communications2020;12. doi:10.1038/s41467-020-20742-8

51 Clifford S, Quilty BJ, Russell TW, et al. Strategies to reduce the risk of SARS-CoV-2 re-introduction from international travellers. medRxiv 2020, doi:10.1101/2020.07.24.20161281 